\title{
Enhancement in the Detection of Atrial Fibrillation Arrhythmia For Health Monitoring System
}

\author{
Sowndarya K, Srinivasan K, Jeevanantham S, Rukkumani V
}

\begin{abstract}
In day to day life, continuous monitoring of medical data is essential for many individuals including patients with severe health risks and elderly people. In this paper, one such system is developed for continuous monitoring of Atrial Fibrillation abnormality. Electrocardiographic (ECG) signal monitoring plays a vital role in the management of patients with atrial fibrillation (AF). Atrial Fibrillation (AF) is a type of abnormality in heart, it causes during the AF electrical discharges in the atrium are rapid and results in an abnormal heartbeat. In this paper, ECG signals taken from the MIT-BIH arrhythmia database. After the signal is acquired, the hybrid filtering technique is used to remove the artifacts. Naturally, the ECG signal gets distorted by different types of artifacts which must be removed from the signal otherwise it will convey incorrect information regarding the patient's heart condition. Efficient LMS and Normalized LMS adaptive filters are computationally used for cancellation of noise. Analyzing functions of the filtered signal is Peak Signal to Noise Ratio (PSNR), Mean Square Normalized Error Performance (MSE), Maximum Square Error (MAXERR), the ratio of Squared Norms (L2RAT). The continuous health statistics will be given to individuals and caretaker in the remote location so that they can take necessary action to prevent from health issues. The paper will provide primitive solutions in the field of telemedicine using continuous health monitoring and medical data analysis of a particular individual.In future work, the ECG sensor will acquire the ECG signal and the acquired signal processed through the classification algorithm for classifying the signal into different categories. The biosensor is a combination of the biological element with the physiochemical transducer to produce an electronic signal which can be further converted, processed and transmitted for data analytics, processing, validation, visualization, interpretation, and data logging.
\end{abstract}

Keywords- MIT-BIH arrhythmia database, Atrial Fibrillation, Health Monitoring, Telemedicine, Data analysis.

\section{INTRODUCTION}

Modern health care technology improves the comfort in the lifestyle of such persons both in physical and mental state. In the medical field, monitoring is considered as the observation of several biological parameters over time, diseases and health condition of an individuals or group of people [1]-[4]. In this field, Electrocardiograph a diagnostic tool deals with the electrical activity of the heart over a period of time by placing the electrode over the skin. The tiny electrical changes arise from the heart muscles will detect on the skin using electrode [17]. The function of cardiac is reflected in the shape of the ECG waveform and heart rate, as well as provides indirect evidence for the blood flow to the heart muscle. Heart rate refers to how fast the heart beats and heart rhythm refers to the type of heartbeat.

Revised Manuscript Received on 14 September, 2019.

Sowndarya K, PG Scholar, Department of Electronics and Instrumentation Engineering, Sri Ramakrishna Engineering College, Coimbatore, Tamil Nadu, India,

Srinivasan K, Professor and Head, Department of Electronics and Instrumentation Engineering, Sri Ramakrishna Engineering College, Coimbatore, Tamil Nadu, India
Normally, the electrical impulse generated by the Sinoatrial node (SA) with the heart beats in a sinus rhythm resulting in a ventricular contraction. The heart is a muscular organ, which functions as a pump for the movement of blood flow through the body by valves [14][15]. The valves function like one-way doors which prevent the backward flow of blood and allow blood flow through the forward direction. The venous blood returns from the body to the right side of the heart which pumps the blood to the lungs [20]-[22]. The oxygen-rich blood returns from the lungs to the left side of the heart and to the entire body. The aortic valve controls the flow of blood out of the heart into the aorta, the largest artery of the body which then gives rise to all the other arteries [8]-[9].

The ECG interpretation traditionally starts with an assessment of the P-wave which reflects atrial depolarization. The PR interval is the distance between the onset of the P-wave to the onset of the QRS complex, determine the impulse conduction from the atria to the ventricles is normal [17]. The flat line between the end of the P-wave and the onset of the QRS complex which reflects the slow impulse conduction through the atrioventricular node is referred as PR segment; it is the baseline of the ECG curve. The QRS complex represents the depolarization of the ventricles and ST segment corresponds to the plateau phase of the action potential [18]-[19]. The T-wave reflects the rapid repolarization of contractile cells and it occurs in a wide range of conditions. The U-wave is a positive wave occurring after the T-wave, which is one-fourth of the T-wave's amplitude and seen occasionally. There are different varieties of arrhythmia, some are normal variants, some are potentially dangerous and some of the cause sudden death without any symptoms. Some of them are Normal sinus rhythm, Sinus tachycardia, Sinus bradycardia, Atrial flutter and so on. Atrial Fibrillation (A F) is one of the most common abnormal heart rhythms, which cause major health problem and $\mathrm{AF}$ shows no $\mathrm{P}$-waves and an irregular ventricular rate [3]. AF is characterized by the rapid and irregular beating of the atria which often have no symptoms [10]. Occasionally there may be symptoms like fainting, shortness of breath, chest pain and high blood pressure, valvular heart disease are some of the common alterable risk factors for AF [11][12].

\section{LITERATURE SURVEY}

[1] Davide Del Testa and Michele Rossi, advocate the use of autoencoders as an efficient and computationally lightweight means to compress biometric signals. While this presented techniques can be used with any signal showing a certain degree of periodicity, in this the technique is applied to ECG traces, showing quantitative results

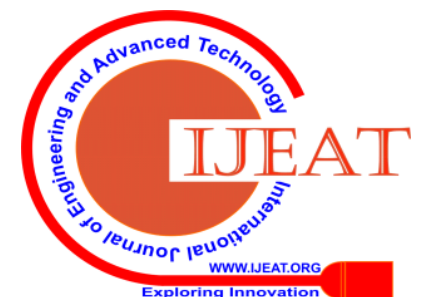


in terms of compression ratio, reconstruction error, and computational complexity, etc [2]. State of the art solutions is also compared with the approach.

[2] JianboGao, Hussain Sultan, Jing $\mathrm{Hu}$, and WenWen Tung propose an effective noise reduction adaptive denoising algorithm with chaotic Lorenz data, root-meansquare-error, Lyapunov exponent and correlation dimension [5]. Further, analyze an electroencephalogram (EEG) signal in sleep apnea and other types of noise-contaminated in EEG than wavelet approaches.

[3] Kameswra Rao P., Bhujanga Rao K., and Anil Kumar B. implement the hybrid algorithm on active noise control which provides better performance than the adaptive technique used to enhance the EEG signal and the fidelity parameters like a signal to noise ratio (SNR), MSE and LSE can be computed [6].

[4] Majid Moavenian, Hamid Khorrami develop a novel use of kernel-Adatron learning algorithm to aid Support Vector Machine (SVM) for ECG arrhythmias classification [7]. In this, the proposed pattern classifier is compared with Multi-layered perceptron using back propagation learning algorithm and the proposed SVM method shows considerable improvement in comparison to reported results.

[5] SasanYazdani, SibylleFallet, and Jean-Marc Vesin propose a fast novel non-linear filtering method named Relative-Energy (Rel-En) for short-term event extraction for biomedical signals [13]. The developed algorithm extracts short and long-term energies in a signal and provides a coefficient vector with which the signal is multiplied, heightening events of interest.

[6] UditSatija, Barathram.Ramkumar and $\mathrm{M}$. SabarimalaiManikandan propose a novel signal quality aware IoT-enabled ECG telemetry system for continuous cardiac health monitoring applications. The proposed quality-aware that the ECG monitoring system consists of three modules: ECG signal sensing module; automated signal quality assessment module; and signal-quality aware ECG analysis and a transmission module [16]. Design and development of a light-weight ECG signal quality assessment method for automatically classifying the acquired ECG signal into acceptable or unacceptable class and real-time implementation of proposed IoT-enabled.

\section{PROPOSED METHODOLOGY}

\section{A. Block Diagram of the Proposed System}

In this proposed work, the performing system has specialized in the following categories. Figure 1 describes the block diagram of the proposed pre-processingsystem. The ECG signal from the human body is measured using ECG sensor, in this paper it is acquired from the database such as MIT-BIH database, Physionet etc., approximately $60 \%$ of these recordings were obtained from inpatients. The database contains 23 records (numbered from 100 to 124 inclusive with some numbers missing) and each of the 48 records is slightly over 30 minutes long. Then the acquired biosignals from the biosignal measurement unit are transmitted to the signal pre-processing and transmission unit, where the hybrid filters process the signal.

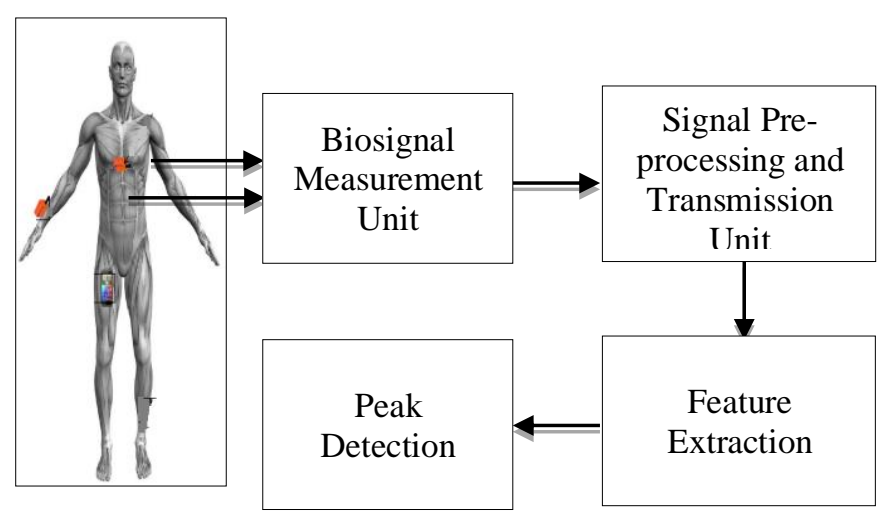

Fig. 1 Block diagram of the proposed pre-processing work

After the pre-processing and transmission unit, the signal passes through feature extraction; the purpose of feature extraction is not only to reduce the dimensionality but also to extract more useful/dominant information hidden in the signals by avoiding unnecessary or redundant information. After features are extracted, the peak of the ECG signal is detected. Pre-processing improves the signal and reduces the noise. Different artifacts affect the ECG signal during its acquisition and transmission. Mainly, there are two types of noises present in the ECG signal. They are noises with high frequency which include Electromyogram noise, Additive white Gaussian noise, and power line interference and noises with a low frequency which include baseline wandering. The noises contaminated in the ECG signal may lead to wrong interpretation.

\section{B. Flow Chart of the Proposed Work}

Figure 2 describes the flowchart of the proposed system. The bio-signals are obtained from the biosensing unit which is the MIT-BIH database and the signal is carried out to the sampling unit for sampling the needed signal. Then it transmits to the pre-processing unit which consists of hybrid filtering. It can be performed through initializing the weight and signal, performing computation with the equation and weight updation. After the completion of pre-processing, the parameter estimation process is performed in which the filtered signal gets analyzed through the analyzing parameters such as PSNR, MSE, MAXERR, and L2RAT then process through the comparison unit 


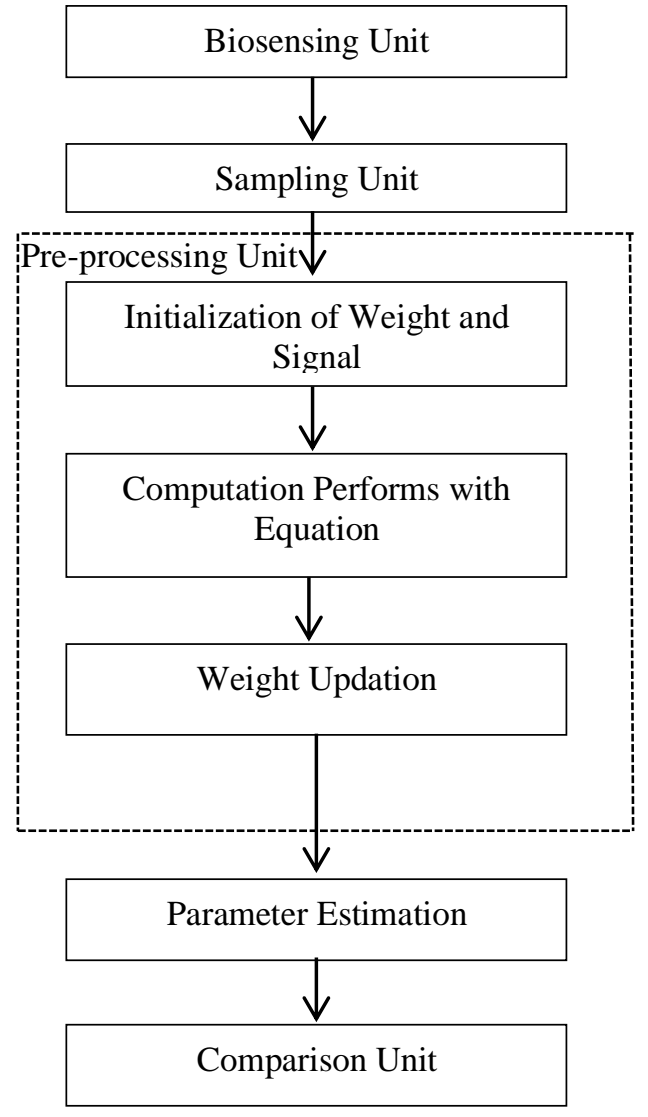

Fig. 2 Flowchart of the proposed system

\section{ALGORITHM DERIVATION}

The proposed hybrid adaptive algorithm is constructed from the Fixed LMS and Normalized LMS algorithms. The detail discusses the two algorithms were given below.

\section{A. Fixed LMS Algorithm}

The mathematical morphology provides an efficient framework for analyzing the Fixed LMS adaptive filters. Mathematical morphology provides the weight update equation for fixed LMS adaptive filter used in ECG signal processing.

The equation of Fixed LMS Algorithm weight update is described in equation 4.1 and 4.2 as

$$
\begin{gathered}
y(n)=w_{0}(n)+w_{1}(n) u(n-1)+\cdots \\
+w_{M-1}(n) u(n-M+1) \\
=\sum_{K=0}^{M-1} w_{k}(n) u(n-k)=w(n)^{T} u(n), \\
n \\
=0,1,2,3, \ldots \infty
\end{gathered}
$$

The error between the filter output $y(t)$ and desired signal $d(t)$ is shown in equation 4.3:

$$
\begin{aligned}
& e(n)=d(n)-y(n) \\
& \quad=d(n)-w(n)^{T} u(n)
\end{aligned}
$$

Change the filter parameters according to equation 4.4

$$
w(n+1)=w(n)+\mu u(n) e(n)(4.4)
$$

\section{B. Normalized LMS Algorithm}

The Normalized LMS Algorithm weight updation is discussed below in equation 4.5. Modify at time $n$ the parameter vector from $\mathrm{w}(\mathrm{n})$ to $\mathrm{w}(\mathrm{n}+1)$ fulfilling the constraint.

$$
\begin{aligned}
& w^{T}(n+1) u(n) \\
& =d(n)
\end{aligned}
$$

With the 'least modification of w(n) i.e., with the least Euclidian norm of the difference is shown in equation 4.6

$$
\begin{array}{r}
w(n+1)-w(n) \\
=\delta w(n+1)
\end{array}
$$

The adjustable weights are typically determined by the LMS algorithm, the weight update equation 4.7 is shown below.

$$
w_{j}(n+1)=w_{j}(n)+\frac{\mu}{|| \mathrm{x}(\mathrm{n})||^{2}} e(n) x(n-f)
$$

\section{RESUlts AND Discussion}

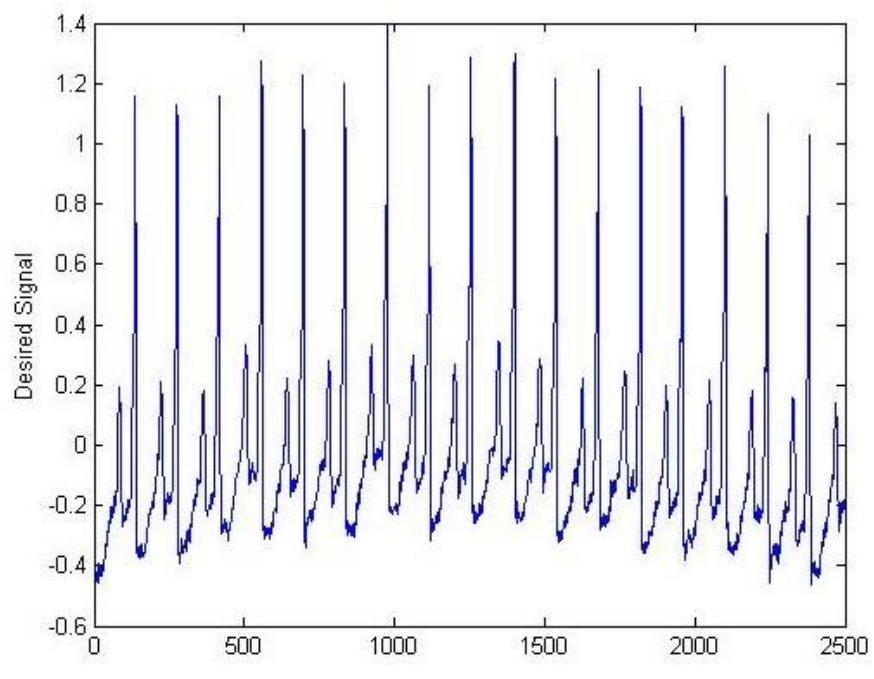

Fig.3Input ECG signal 1

Atrial Fibrillation has no $\mathrm{p}$ wave and the second wave is the QRS complex. Typically this reflects the current associated with right and left ventricular depolarization with a series of 3 deflections. If the first deflection in the complex is negative, is called a $\mathrm{Q}$ wave. The first positive deflection in the complex is known as an $\mathrm{R}$ wave. A negative deflection after an $\mathrm{R}$ wave is referred to as an $\mathrm{S}$ wave. A second positive deflection after the $S$ wave is named as the Rwave. In this work, the ECG signal is acquired from the database such as the MIT-BIH database, Physionet etc. The LMS algorithm is robust in nature, slow in convergence and sensitive to variations in step size which also requires number of iterations equals to dimensionality of the input. In NLMS algorithm, the estimated error value between the desired signal and filter output will be less, the convergence also occur faster. 


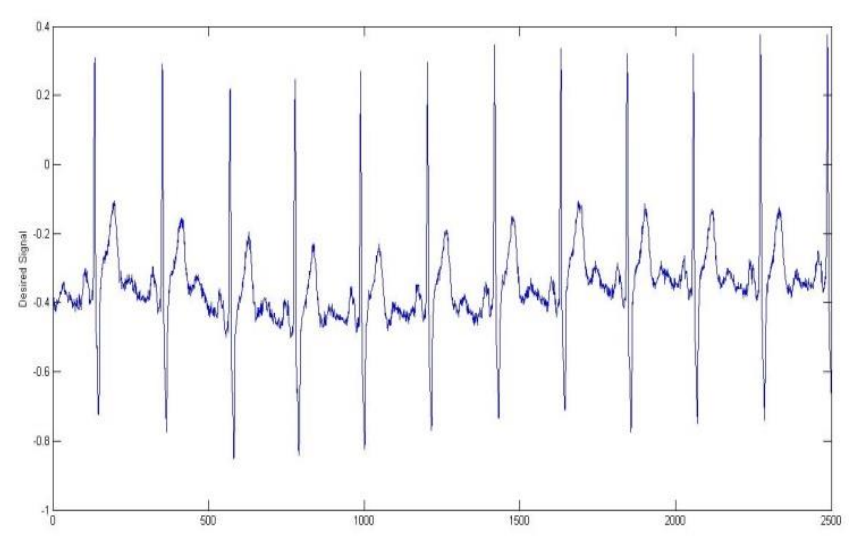

Fig. 4 Input ECG signal 2

By combining these algorithms the sensitive to variations in step size will be reduced, the algorithm will be more stable, convergence will be high and less complex. Figure 3and 4 show the input ECG signal 1 and 2. Figure 5and 6 show the desired output of the hybrid filter for input signal 1 and 2, which is the combination of LMS and Normalized RLMS.

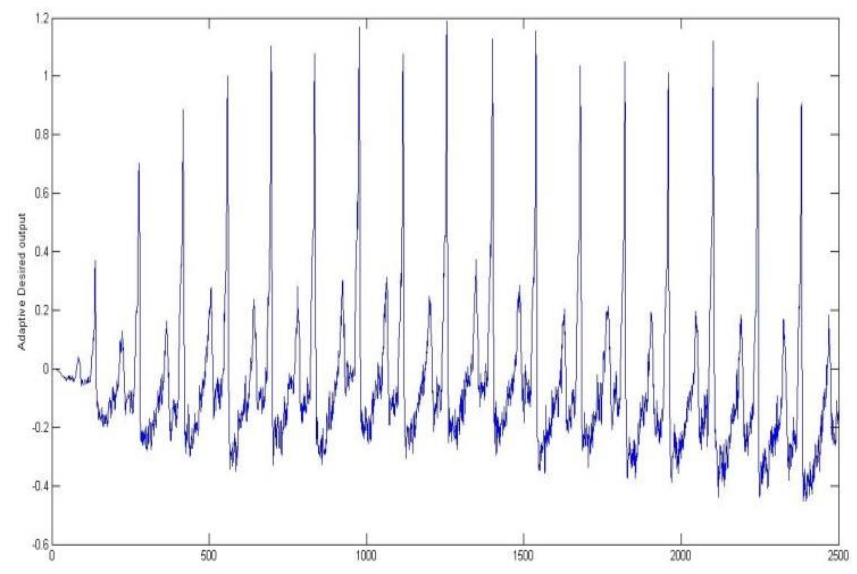

Fig. 5Output of the hybrid filter for input signal 1

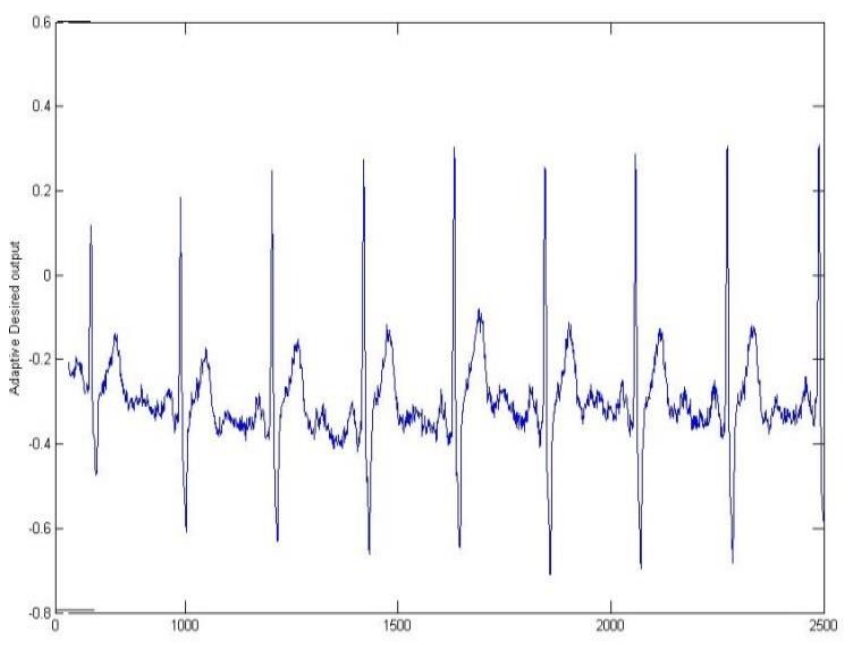

Fig. 6 Output of the hybrid filter for input signal 2

Analyzing functions of the filtered signal PSNR, MSE, MAXERR, and L2RAT.Peak signal-to-noise ratio, often abbreviated PSNR, is an engineering term for the ratio between the maximum possible power of a signal and the power of corrupting noise is described in equation 5.1. The mean squared error (MSE) of an estimator measures the average of the squares of the errors, the average squared difference between the estimated values and what is estimated is expressed in equation 5.2.

Table 1 Quality metrics of the filtered signals

$P S N R=10 \log _{10} \frac{M A X_{i}^{2}}{M S E}$

\begin{tabular}{|l|c|c|c|}
\hline \multicolumn{5}{|c|}{ Signal 1 } \\
\hline $\begin{array}{l}\text { Analyzing } \\
\text { functions }\end{array}$ & LMS & NLMS & Hybrid \\
\hline PSNR & 60.15 & 56.41 & 66.45 \\
\hline MSE & 0.06 & 0.15 & 0.014 \\
\hline MAXERR & 1.10 & 1.42 & 0.49 \\
\hline L2RAT & 0.23 & 0.87 & 0.14 \\
\hline \multicolumn{4}{|c|}{ Signal 2} \\
\hline $\begin{array}{l}\text { Analyzing } \\
\text { functions }\end{array}$ & LMS & NLMS & Hybrid \\
\hline PSNR & 58.59 & 63.07 & 63.21 \\
\hline MSE & 0.09 & 0.02 & 0.03 \\
\hline MAXERR & 1.05 & 0.88 & 0.70 \\
\hline L2RAT & 0.25 & 0.95 & 0.35 \\
\hline
\end{tabular}

Table 1 shows the comparison between LMS, NLMS and the hybrid algorithm with the help of analyzing functions. From the above table, it is concluded that the analyzing function values are most efficient for the hybrid than LMS and NLMS filtering technique.Figure 7 shows the comparison chart for LMS, NLMS and Hybrid filter with PSNR value. From the graph, it is concluded that the output of the hybrid filter is more efficient than the LMS and NLMS filter.

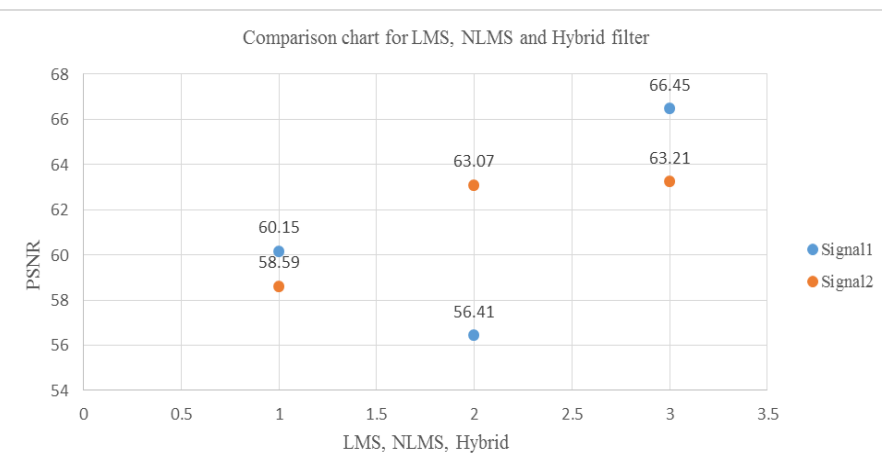

Fig. 7 Comparison chart for LMS, NLMS and Hybrid filter with PSNR value

\section{CONCLUSION AND FUTURE SCOPE}

In clinical practice, the ECG signal is the most widely used for recording electrical signal from the heart. By altering the shape of its constituent waves, namely the P, QRS, and T waves ECG conveys information regarding the electrical function of the heart. In this paper, the Hybrid algorithm is developed with LMS and Normalized LMS for providing more stable, step size, less compact and most reliable results are obtained for the input 
signal. In future work, the ECG signal can acquire from the ECG sensor and the acquired signal can be processed through the algorithm which is used for classifying the ECG signal into three different types: Paroxysmal, Persistent and Permanent. In Paroxysmal condition, the AF will occur sometimes and then stops. During persistent conditions, the meditations or a special type of electrical charge is required. In Permanent condition, the normal rhythm cannot occur.In future, the data received from the ECG sensor will be displayed and stored in the individual data path and the above data will be transmitted to remote user and processing server through the Internet of Things (IoT) platform which forms real-time function.

\section{REFERENCES}

1. BabakMohammadzadehAsl, SeyedKamaledinSetarehdan, Maryam Mohebbi, "Support vector machine-based arrhythmia classification using reduced features of heart rate variability signal," Artificial Intelligence in Medicine, vol. 44, no. 1, pp. 51-64, April 2008.

2. Davide Del Testa and Michele Rossi, "Lightweight lossy compression of biometric patterns via denoisingAutoencoders," IEEE Signal Processing, vol. 22, no. 12, pp. 2304-2308, December 2015.

3. David D. McManus, Jinseok Lee, Oscar Maitas, Nada Esa, Rahul Pidikiti, Alex Carlucci, Josephine Harrington, Eric Mick, Ki H. Chon, "A novel application for the detection of an irregular pulse using an iPhone 4S in patients with atrial fibrillation," Science Direct- Heart Rhythm, vol. 10, no. 03, pp.-315-319, March 2013.

4. Daniel Scherr, DarshanDalal, Aamir Cheema, Alan Cheng, Charles A. Henrikson, David Spragg, Joseph E. Marine, Ronald D. Berger, Hugh Calkins, Jun Dong, "Automated detection and characterization of complex fractionated atrial electrograms in human left atrium during atrial fibrillation," Science Direct- Heart Rhythm, vol. 04, no. 08, pp. 1013-1020, May 2007.

5. Devasena D., Jagadeeswari M., "FPGA implementation of speckle noise removed in real time medical images," Journal of Medical Imaging and Health Informatics, vol. 7, pp. 1-8, October 2017.

6. Jianbo Gao, Hussain Sultan, Jing Hu, and Wen-Wen Tung, "Denoising Nonlinear Time Series by adaptive filtering and wavelet shrinkage: a comparison," IEEE Signal Processing, vol. 17, no. 3, pp. 237 240,March 2010.

7. Kameswra Rao P., Bhujanga Rao A., Anil Kumar B., "DE-Noising of ECG signal using hybrid adaptive filters," International Journal for Research in Applied Science \& Engineering Technology, vol. 5, no. 12, pp. 721-727, December 2017.

8. Majid Moavenian, Hamid Khorrami (2010), “A qualitative comparison of artificial neural networks and support vector machines in ECG arrhythmias classification," Expert Systems with Applications vol. 37, no. 4, pp. 3088-3093, April 2010.

9. Marco Tomasini, Simone Benatti, Bojan Milosevic, ElisabettaFarella Luca Benini, "Power line interference removal for high quality continuous bio-signal monitoring with low-power wearable devices,'IEEE Sensors Journal, vol. 16, no. 10, pp. 3887 - 3895, May 2016.

10. Mora F.A., "Intelligent patient monitoring and management systems: a review," IEEE Engineering in Medicine and Biology Magazine, vol. 12, no. 4, pp. 23-33, December 1993.

11. NicosMaglaveras, TelemachosStamkopoulos, Konstantinos Diamantaras, Costas Pappas, Michael Strintzis, "ECG pattern recognition and classification using non-linear transformations and neural networks: A review," International Journal of Medical Informatics, vol. 52, pp.191-208, October 1998.

12. PriyaDharshini K., Ramya R., Srinivasan K., "Certain investigation on prenatal medical image analysis," International Conference on Electrical, Instrumentation and Communication Engineering, pp. 1-5, December 2017

13. RahimeCeylan, YukselOzbay, "Comparison of FCM, PCA and WT techniques for classification ECG arrhythmias using artificial neural network," Expert Systems with Applications, vol. 33, no. 2, pp. 286295, August 2007.

14. Ramya R., Srinivasan K., Sharmila B., PriyaDharshini K.,"Feature Selection in Fetal Biometrics for Abnormality Detection in Ultrasound Images,"Nature Inspired Optimization Techniques for Image Processing Applications, vol. 150,pp 279-297, September 2018.

15. Saeed Babaeizadeh, Richard E. Gregg, Eric D. Helfenbein, James M. Lindauer, Sophia H. Zhou, "Improvements in atrial fibrillation detection for real-time monitoring," Journal of Electrocardiology, vol. 42, no. 6, pp. 522-526, December 2009.

16. SasanYazdani, SibylleFallet, Jean-Marc Vesin, "A novel short-term event extraction algorithm for biomedical Signals," IEEE Transactions on Biomedical Engineering, vol. 65, no. 4, pp. 754 - 762, April 2018.

17. SucharitaMitra, MadhuchhandaMitra, Chaudhui B.B., "A Rough-SetBased inference engine for ECG classification," IEEE Transactions on Instrumentation and Measurement, vol. 55, no. 6, pp. 2198 - 2206, December 2006.

18. Sowndarya V., Tamilselvi V., Srinivasan K., Rukkumani V., "Smart jacket for the coal miners based on wireless sensor networks and WoT," International Journal on Applications in Information and Communication Engineering, vol. 3, no. 1, pp. 12-17, January 2017.

19. Tsipouras M.G., Fotiadis D.I., Sideris D., "An arrhythmia classification system based on the RR-interval signal," Artificial Intelligence in Medicine, vol. 33, no. 3, pp. 237-250, March 2005.

20. UditSatija, Barathram.Ramkumar, and M. SabarimalaiManikandan, "Real-Time signal quality-aware ECG telemetry system for IoT-Based health care monitoring," IEEE Internet of Things, vol. 4,no. 3, pp. 815-823, June 2017.

21. Wei Jiang, Seong G. Kong (2007), "Block-Based neural networks for personalized ECG signal classification," IEEE Transactions on Neural Networks, vol. 18, no. 6, pp. 1750 - 1761, November 2007.

22. Yu Hen Hu, Surekha Palreddy, Willis J. Tompkins, "A patientadaptable ECG beat classifier using a mixture of experts approach," IEEE Transactions on Biomedical Engineering, vol. 44, no. 9, pp. 891 900, September 1997. 